\title{
Limits of composition achievable by ion implantation
}

\author{
Z. L. Liau and J. W. Mayer \\ California Institute of Technology, Pasadena, California 91125
}

(Received 31 August 1978)

In high-dose ion implantation for materials modification, the maximum concentration of the implanted species is determined by ion-induced erosion (sputtering) of the implanted layer. In this review, we consider the influence of preferential sputtering and atomic mixing. The maximum concentration of the implanted species is given roughly by $r / S$ and extends over a depth $W$ where $S$ is the sputtering yield, $r$ is the preferential sputtering factor $(1 / 2 \lesssim r \lesssim 2)$ and $W$ is a depth comparable to the ion range. Good agreement between calculation and experiment is found for $150-\mathrm{keV} \mathrm{Au}$ implanted into $\mathrm{Cu}$ or $\mathrm{Fe}$. Surface conditions, such as oxide layers or carbon films, can alter sputtering yields and can lead to the mixing of surface contaminants throughout the implanted layer. Implantation of species $A$ into a target material $A B$ results in a different concentration limit, but again preferential sputtering and the total sputtering yield set this limit. Calculations for PtSi indicate that the concentration of $\mathrm{Si}$ is decreased by implantation of $\mathrm{Si}$ for $S>3$.

PACS numbers: $61.70 . \mathrm{Tm}$

\section{INTRODUCTION}

The impact of ion-implantation in semiconductor device technology is due to external control of the number of ions per $\mathrm{cm}^{2}$ implanted in the device. Along with the concept of dose control came the possibility of dose reproducibility from wafer to wafer in the production line. The idea that in doping of semiconductors one could be independent of surface conditions or the chemistry at the gas-solid interface had a very large appeal to those producing semiconductor devices. All they had to measure was a current of ions.

Coupled with the attractiveness of implantation was the large number of developments in the analytical evaluation of sub-micron regions in solids ${ }^{1-4}$. There have been improvements in nuclear analysis techniques such as backscattering and channeling and in sputter profiling techniques such as secondary ion mass spectrometry (SIMS) and Auger electron spectrometry (AES). These analytical tools allowed one the opportunity to explore the properties of implanted layers. These techniques enabled rapid strides be made in the understanding of the implantation process.

In another field, metals, there have been major developments in implantation metallurgy. It has now been demonstrated in practical devices and in laboratory research that implantation into solids can have striking effects in wear resistance, corrosion resistance, and in production of unusual metastable alloys ${ }^{5,6}$. Many of the papers in the present conference reflect some of these advances in ion beam modification of materials.

There has been one dramatic change in the parameters applied to implantation as the field has moved from semiconductors to metals: the dose has been increased by orders of magnitude. Even in semiconductor devices with high doping concentrations on the order of 0.1 at. \%, the ion doses are moderate, of the order of $10^{15}$ ions $\mathrm{cm}^{-2}$ or less. In implantation metallurgy, one introduces concentrations of im- planted ions of a few atomic percent corresponding to implanted doses of about $10^{16}$ ions $\mathrm{cm}^{-2}$.

The thrust of the present paper is the concentration limit that can be achieved with ion implantation, at an order of magnitude higher dose of implanted ions $\left(\gtrsim 10^{17}\right.$ ions $\left.\mathrm{cm}^{-2}\right)$ in order to achieve concentration of implanted species of greater than 10 at. \%. The central problem is sputtering, in which the transfers of energy from the incident ion to the material results in the ejection of atoms from the surface. ${ }^{7-9}$ For a dose of $10^{17}$ ions $\mathrm{cm}^{-2}$ (about 100 monolayers) it is possible to remove from 100 to 1000 layers of the target material, i.e., from 500 to $5000 \AA$.

As indicated in the sketch in Fig. 1 there is a pronounced change in the distribution of impurities. In the upper part of the figure for low-dose implantation, the implanted atoms are located at a depth within the sample given by the range of the ions, $R_{p}$ and by the spread in the ion distribution. In the lower part of Fig. 1, where the sample surface is eroding during implantation, the resultant profile is due to erosion and sputtering of both the target and the implanted ions. The simplest estimate ${ }^{10}$ gives the concentration of implanted species to be proportional to $1 / S$, where $S$ is the sputtering yield or number of target atoms removed for one incident ion.

Recent developments in experiments on sputtering of compounds have indicated that preferential sputtering effects can dominate. ${ }^{11-16}$ The influence of preferential sputtering is generally to reduce the concentration of lighter mass atoms at the surface of the target. Due to the atomic mixing that occurs along the track of the incident ion beam, this depletion of lower mass ions from the target surface will be spread throughout the target over the depth of the implanted distribution. The purpose of the present paper then is to explore the limits of concentration achievable by implantation when one takes into account preferential sputtering and atomic mixing. Other topics dealing with precipitation and com- 

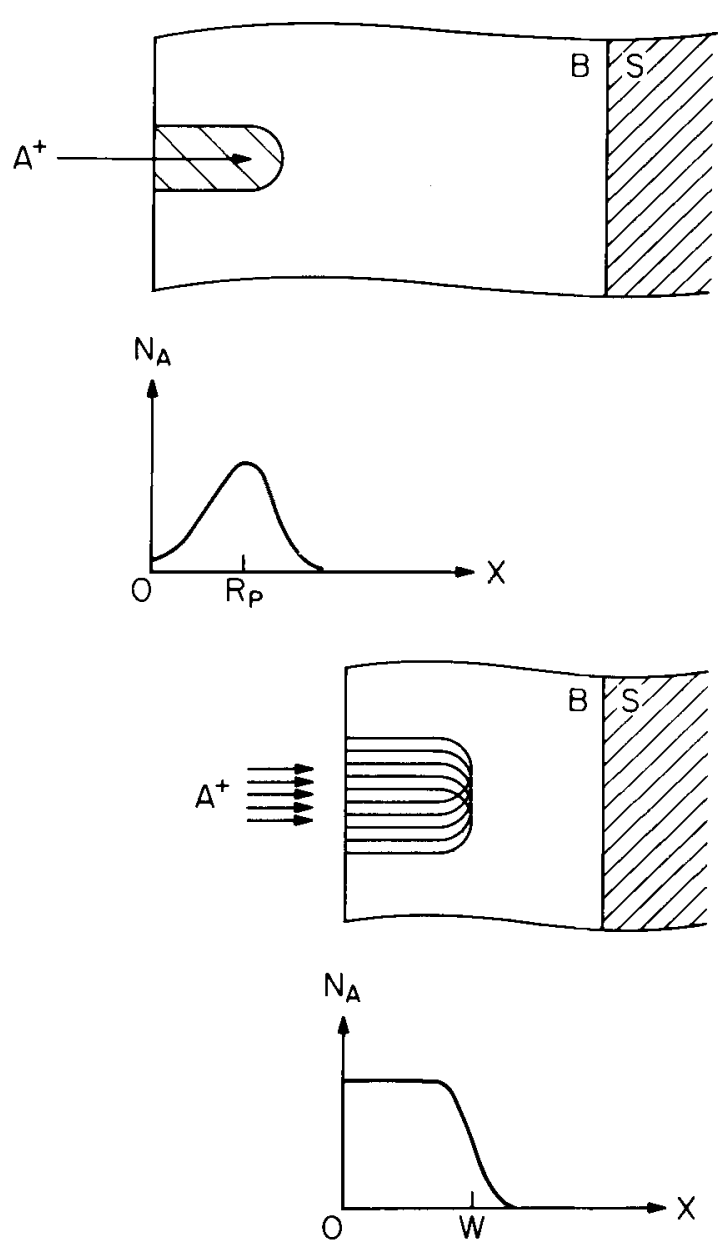

FIG. 1. A schematic drawing which illustrates effects of high-dose ion implantation of ions $A$ into material $B$, depicted as a thin film on a substrate $S$. In the low-dose region, the effects of atomic mixing and sputtering are important in determining the state of implanted material.

pound formation will be discussed in other papers presented at this conference.

\section{BACKGROUND ON SPUTTERING}

The sputtering of monoelemental solids exhibits good agreement between theory and experiment. ${ }^{17}$ Figure 2 shows the sputtering yield $S$ for $\mathrm{Si}, \mathrm{Cu}$, and $\mathrm{Au}$ as a function of the atomic number $Z_{1}$ of the $45-\mathrm{keV}$ incident ions. In order to construct this plot we have taken relative sputtering yields ${ }^{18,19}$ and adjusted them for the sputtering yield of $45-\mathrm{keV} \mathrm{Ar}$ ions incident on the different targets. The solid lines represent theoretical predictions of Sigmund ${ }^{17}$ One can see that for low values of the atomic number of the host target there is very good agreement between the experimental and theoretical values of the sputtering yield. The deviations between theory and experiment are most pronounced for high-mass ions in gold where the sputtering yield is higher than the theoretical prediction. It has recently been shown that this enhanced sputtering yield is due to the high amount of energy deposited within the collision cascade around the track of the incident ion. ${ }^{19}$ Sputtering of elemental targets is well understood as long as the dose of the implanted species is not sufficiently high so that the implanted concentration exceeds a few atomic percent.

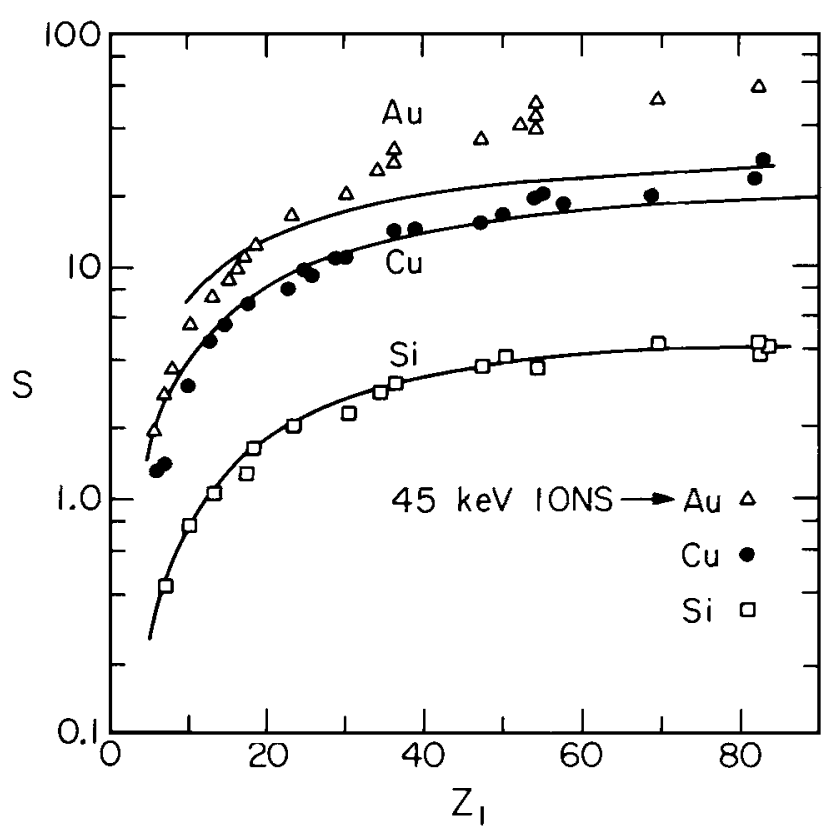

Fig. 2. Sputtering yields of $\mathrm{Au}, \mathrm{Cu}$, and $\mathrm{Si}$ by $45-\mathrm{keV}$ ions of various atomic numbers, $Z_{1}$. The solid lines are calculations due to Sigmund (Refs. 18 and 19).

Sputtering of multielements samples is not as well understood. One observes that it is not possible to simply calculate the sputtering yield of elements from the sample from the sputtering yield of the same elements in the monoelemental sample. This was demonstrated in a series of measurements of the sputtering yields of compounds and of monoelemental targets of the same materials. ${ }^{12,14}$ Figure 3 shows the backscattering spectra for platinum silicide samples sputtered by 900-eV Ar ions. ${ }^{12}$ The spectra show at the high energy edge region between 1.4 and $1.8 \mathrm{MeV}$ the signals from the Pt atoms in the Pt-silicide target, indicated by PtSi. At the low-energy end from about $0.8 \mathrm{M}$ to $1.1 \mathrm{MeV}$ one has the backscattering

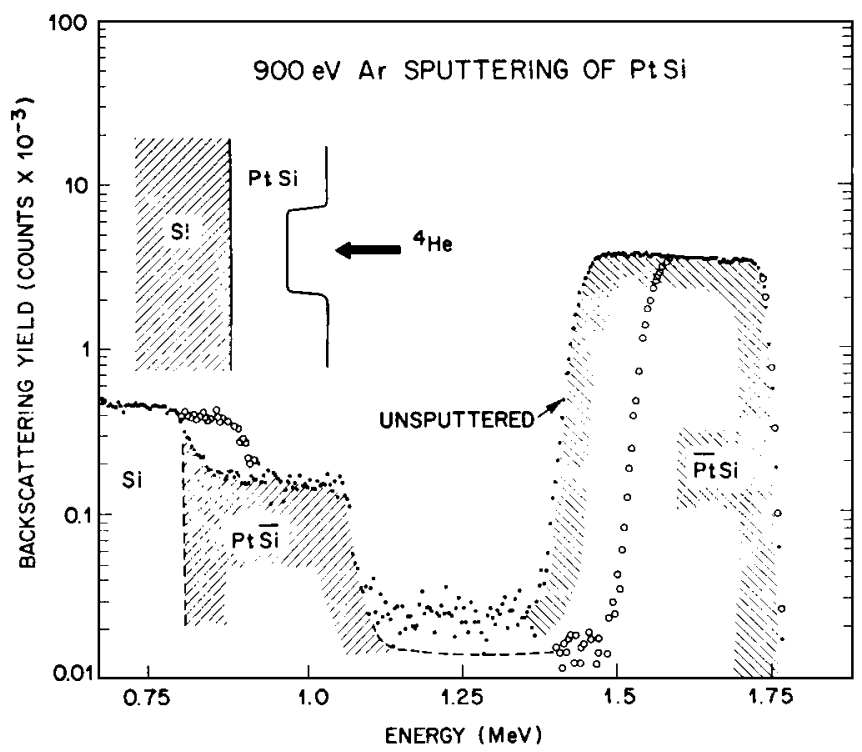

FIG. 3. Measurement of the sputtering yield of a compound. This figure shows backscattering spectra of a PtSi film before and after being sputtered by $900-\mathrm{eV} \mathrm{Ar}^{+}$. The sputtering yield is obtained by measuring the change of the PtSi film thickness versus Ar ion dose (Ref. 12). 


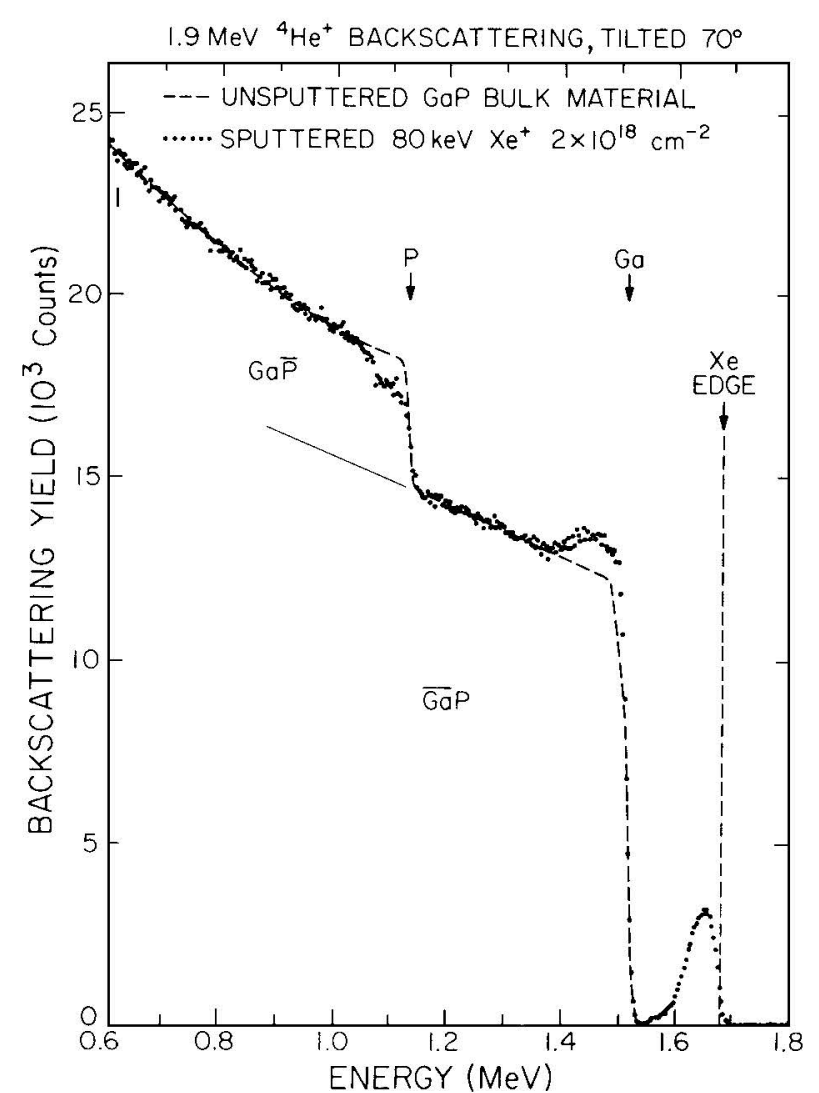

FIG. 4. Backscattering spectrum of a GaP sample after being sputtered by $80-\mathrm{keV} \mathrm{Xe}^{+}$and that of an unsputtered one. The sputtered sample shows a Ga enrichment in a surface layer of $\sim 500 \AA$ (Ref. 20 ).

yield from Si atoms in $\mathrm{Pt}$-silicide indicated by $\mathrm{Pt} \overline{\mathrm{Si}}$. From the difference in the two spectra representing the unsputtered and sputtered samples, one can determine both the amount of $\mathrm{Pt}$ and the amount of $\mathrm{Si}$ that have been removed or sputtered from the target. From measurements such as these one finds that the sputtering yield of $20-\mathrm{keV}$ Ar ions, for elemental $\mathrm{Pt}$ is 4.1, for elemental $\mathrm{Si}$ is 1.5 , and for the total yield $S$ of Pt-silicide is $4.7 .^{12}$

The sputtering of multielemental targets also shows conclusively that there is preferential sputtering of the light elements within the target. ${ }^{11-16}$ The data in Fig. 4 show the spectrum of an 80-keV Xe-sputtered GaP sample and that of an unsputtered one. ${ }^{20}$ The backscattering spectrum of the sputtered target indicates that the process of sputtering has depleted phosphorus and has increased the gallium content in the sputtered layer. This phenomenon has been seen in oxide layers, miscible compounds, metallic compounds and silicides as well as in the present example of a compound semiconductor ${ }^{13}$ The ratio $r$ of the probabilities of sputtering a light ion to that of a heavy ion range from values of 1.1 for targets such as $\mathrm{Au}-\mathrm{Cu}$ to values of 2 for targets such as PtSi. For the case of PtSi this leads to a surface layer that has a 2:1 $\mathrm{Pt}$ to $\mathrm{Si}$ ratio and for the case of $\mathrm{GaP}$ shown in Fig. 4 the ratio of $\mathrm{Ga}$ to $\mathrm{P}$ is about 1.5:1.

At the present time there have been a number of theories advanced to account for preferential sputtering. These theories are based on factors such as differences in the amount of energy transferred to heavy or light ions, differences in the range of the heavy or light ions or differences in binding energies. At the present time there have not been enough experimental tests to indicate which of the various theories can be used to predict the sputtering yields of multielemental targets. In other words, the present situation is that one must actually perform a sputtering measurement on a multi-elemental target in order to predict the general behavior of sputtering in this type of target.

Preferential sputtering by itself would not be a serious factor if the change in the composition of the target only occurred on the outer most layers of the target, where most sputtered atoms emerge from. In the sputtering process the energy deposited in the target around the path of the ion leads to a high energy density within the collision cascade. This high energy density leads to atomic mixing, that can occur in the relatively short time $\left(10^{-12} \mathrm{~s}\right)$ in which the energy is relaxing to thermal values or afterward as a defect- or radiation-enhanced diffusion. Over depths slightly in excess of the range of the implanted species, there is a large amount of atomic mixing which transfers the change in composition in the surface of the sample due to preferential sputtering inward to depths corresponding to the range of the sputtering ion. This can be seen for example in Fig. 4 for GaP where one sees that the Ga enrichment occurs over the depth distribution of the implanted Xe.

Another aspect of atomic mixing can be found in cases where silicides are formed by ions penetrating through a metallic layer into a silicon substrate. ${ }^{21,22}$ In these investigations, a metal film of $\mathrm{Pd}$, Pt or other silicide former is deposited on a silicon substrate and then the sample is bombarded with ions. It has been found that silicide formation does not occur as long as the range of the implanted atom is less than the thickness of the metal layer. Only when the ion has sufficient energy to penetrate through the interface between the metal layer and the silicon substrate does silicide formation occur during bombardment.

Other evidences for atomic mixing around the track of an incident ion are evidenced in precipitation effects, such as formation of AlSb precipitates in Sb-implanted $\mathrm{Al}^{23}$ or formation of Ar bubbles in Ar-implanted silicon. ${ }^{24} \mathrm{~A}$ dramatic case of Ar bubble formation has been found in the case of $\mathrm{Ar}$ sputtered Pt-silicide. ${ }^{25}$ Figure 5 shows a transmission electron micrograph of Pt-silicide sample sputtered by $160-\mathrm{keV} \mathrm{Ar}$

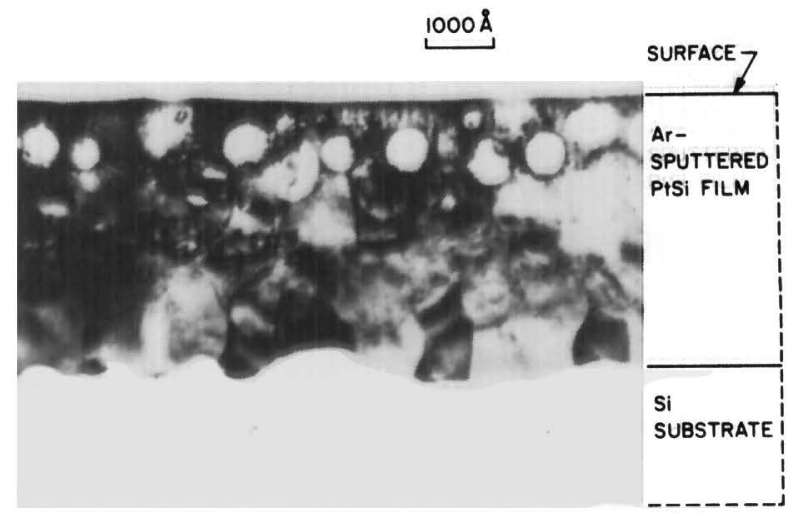

FIG. 5. A TEM photo which shows the cross-sectional view of a PtSi film after being sputtered by $160-\mathrm{keV}$ Ar. The circular regions indicate Ar bubble formation due to high-dose implantation of Ar. The Ar dose was $6.6 \times 10^{16}$ $\mathrm{cm}^{-2}$ (Ref. 25). 
ions. In this transverse view the circular objects in the micrograph represent the Ar bubbles contained within the $\mathrm{Pt}$-silicide. Our view of this process is that during the sputtering process the Ar ions deposit energy along their track causing enhanced diffusion throughout the collision cascade. Any Ar ions within one of the collision cascades tends to migrate to the nucleation site and an Ar bubble is formed. During prolonged bombardment the number of atoms trapped at the bubble continues to grow.

\section{HIGH-DOSE IMPLANTATION INTO ELEMENTAL TARGETS}

\section{A. Model}

In order to develop ${ }^{16}$ a simple model to predict the maximum concentration of implanted species in an elemental target we shall ignore some real experimental effects such as precipitation and cone formation and assume that a uniformly dispersed atomic mixture is formed. We also assume that atomic mixing is very efficient such that the implanted species is distributed uniformly in depth over a width $W$ as shown in Fig. 6. Under these assumptions the shape of the profile remains unchanged, but the amplitude increases with increasing dose until it reaches the steady-state value.

The conservation of atoms require that

$$
W d N_{A} / d t=J_{i}-J_{A}
$$

where $N_{A}$ is the concentration of implanted species, $J_{i}$ is the flux of incident ions (species $A$ ), and $J_{A}$ is the flux of species A sputtered back from the target. At steady state, there is no change in the total number of $A$ atoms in the material, so that

$$
J_{A}=J_{i} \text {. }
$$

We define the total sputtering yield $S$ as

$$
\left(J_{A}+J_{B}\right)=S J_{i}
$$

where $J_{B}$ is the flux of $B$ atoms being sputtered from the target. In terms of $J_{i}$, the term $J_{B}$ can be written

$$
J_{B}=(S-1) J_{i} .
$$

At this point we deviate from the standard approach and introduce the concept of preferential sputtering. We assume that species A and B have a different probability to be sputtered.

$$
J_{B} / J_{A}=r\left(N_{B} / N_{A}\right)
$$

where $r$ is the ratio of the probability for a $B$ atom near the surface to be sputtered to that of an $A$ atom near the surface.

The steady state, or maximum achievable concentration, can then be given as

$$
N_{A} / N_{B}=r /(S-1)
$$

The concentration ratio is roughly inversely proportional to the sputtering yield $S$, but multiplied by the preferential sputtering factor $r$. For example, if the probability of sputtering $B$ is greater than that for $A(r>1)$, the concentration

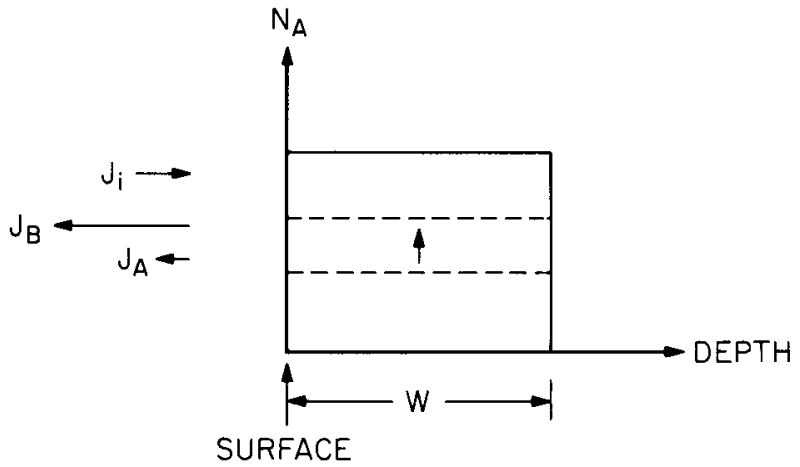

FIG. 6. A schematic picture illustrating the model for calculating implanted concentrations in the high dose region. Along with the increase of the implanted concentration $N_{A}$, the flux of sputtered $A$ atoms, $J_{A}$, increases and approaches that of the incident ions (of species $A$ ), $J_{i}$. The system then approaches a steady state in which there is no further increase in $N_{A}$. Based on this model the steady state is reached with after a sputter-removal of a thickness $\sim r W$, where $r$ is the preferential sputtering factor defined in Eq. (6) and $W$ is the thickness of the implanted layer.

of $A$ atoms would be higher than that in the absence of preferential sputtering.

\section{B. Examples}

As an example of the calculation we show two cases for sputtering by $150-\mathrm{keV}$ Au ions. ${ }^{26}$ Backscattering measurements were used to determine $S$ and $W$. The values of $r$ were assumed to be unity in both cases although for the $\mathrm{Au}-\mathrm{Cu}$ system, alloy sputtering data indicated that the value for $r$ should be 1.1 rather than unity. ${ }^{13}$

The data in Fig. 7 shows the ratio of the concentration of the implanted species Au to the concentration of the elements in the host lattice versus the thickness of the sputter removed layer divided by $W$. The solid lines represent the calculated curve based on the model presented in the previous section. The experimental points were determined by backscattering spectrometry. As indicated in the figure, there is good agreement between the calculated curve and the experimental data. The calculations were based on the two experimentally determined parameters, the total sputtering yield $S$ and the width of the implanted layers $W$. The difference in the maximum concentration of implanted $\mathrm{Au}, 0.05$ for $\mathrm{Cu}$ and 0.3 for $\mathrm{Fe}$, represents the difference in the sputtering yield between the two materials.

\section{PREDICTION OF STEADY-STATE CONCENTRATION.}

In this section we compare the difference in maximum concentrations that can be obtained by implanting $\mathrm{Pt}$ into $\mathrm{Si}$ and that for implanting Si into Pt films.

To obtain the concentration of $\mathrm{Si}$ in Pt that can be obtained by implantation of $45-\mathrm{keV} \mathrm{Si}$ ions, we must first determine the sputtering yield. The sputtering yield of Pt by $20-\mathrm{keV} \mathrm{Ar}$ has been measured to be $4.1 .^{12}$ From the measurements of the sputtering yield of Au by Ar of various energies, the sputtering yield of $\mathrm{Pt}$ should be multiplied by a factor of 1.1 when going from 20 to $45 \mathrm{keV}$; therefore, the sputtering yield of $45-\mathrm{keV}$ Ar into Pt can be estimated to be about 4.5. To determine the 


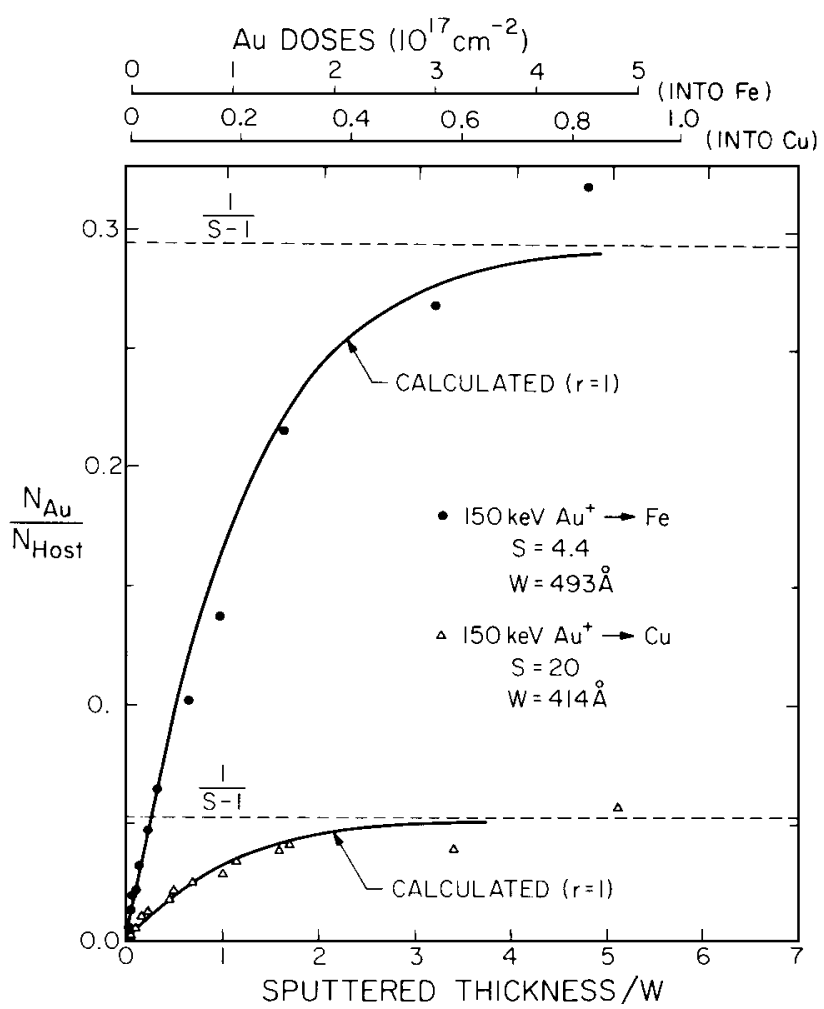

FIG. 7. Buildup of Au concentrations in the implantations of $\mathrm{Au}$ into $\mathrm{Fe}$ and $\mathrm{Cu}$. The $\mathrm{Au}$ concentration in $\mathrm{Cu}$ shows a saturation value of $\sim 5 \%$, which is much lower than that in $\mathrm{Fe}$. This is because the sputtering yield of $\mathrm{Cu}$ is much higher than that of $\mathrm{Fe}$. The curves are calculated from the simple model (Fig. 6) using the experimentally measured $S$ and $W$ (Ref. 26).

sputtering yield of $\mathrm{Pt}$ by $\mathrm{Si}$ ions, we again use Au data from Fig. 2 and note that the sputtering yield of $\mathrm{Au}$ for $\mathrm{Si}$ ions is about $2 / 3$ that for Ar ions. Hence, the total sputtering yield of Pt by $45-\mathrm{keV}$ Si can be estimated to be about 3.0 . In Pt-silicide sputtering, measurements have shown that $\mathrm{Si}$ is preferentially sputtered. Consequently, the value for $r$ in this case is 0.5 . For values of the sputtering yield $S$ equal to 3.0 and $r$ equal to 0.5 , Eq. (6) indicates that the maximum surface concentration ratio is

$$
N_{\mathrm{Si}} / N_{\mathrm{Pt}}=0.25 \text {. }
$$

A sputter-removal thickness of about $320 \AA$ is required to reach the steady state. This occurs at a Si dose of about $0.7 \times$ $10^{17}$ ions $\mathrm{cm}^{-2}$. 16

To determine the concentration of $\mathrm{Pt}$ that can be obtained by sputtering or implanting $\mathrm{Si}$ with $45-\mathrm{keV} \mathrm{Pt}$ we estimate the sputtering yield from the data in Fig. 2 to be about 4.5. Again in the $\mathrm{Pt}$-silicide case where preferential sputtering has been observed, we have a value of $r=2$. Inserting a value of $S=$ 4.5 and $r=2.0$ in Eq. (6), we predict the steady-state surface concentration ratio $N_{\mathrm{Pt}} / N_{\mathrm{Si}}$ to be 0.57 . The thickness that has to be sputtered away is about $1100 \AA$ and the Pt dose of $1.3 \times$ $10^{17}$ ions $\mathrm{cm}^{-2}$ is required. ${ }^{16}$

The factor of two difference in the maximum attainable concentration ratio is due to the preferential sputtering of $\mathrm{Si}$. In the example shown in the previous section for $150-\mathrm{keV} \mathrm{Au}$ ions implanted into $\mathrm{Fe}$ and $\mathrm{Cu}$ the difference in the obtainable concentration was primarily based on the difference in total sputtering yield $S$. Consequently, to predict maximum achievable concentrations it is necessary to have a good measure of the sputtering yield $S$ and the preferential sputtering parameter $r$.

\section{THE INFLUENCE OF OXIDE LAYERS OR CARBON COATINGS}

In order to overcome the limitations set by the sputtering yield, it is attractive to consider the possibility of introducing a surface coverage of carbon or oxide that can suppress the sputtering of the substrate. As sputtering occurs from the outer most layers of the sample, one would anticipate that the surface coatings would be sputtered and the substrate protected. Consider the possibility of performing a high-dose implantation with a high local concentration of oxygen in the vicinity of the sample surface. An oxide layer would build up and if the implanted species tended to be rejected from the oxide layer, high concentrations could be achieved.

Although the concept of the use of surface coatings is attractive on the basis of achieving high concentration of implanted species, it has a serious drawback in that the elements contained in the surface coating will be mixed within the implanted layer. Atomic mixing will cause a redistribution of the surface elements throughout the implanted layer. This effect has been studied for cases of implantation through oxide layers or in oxygen ambients. 27,28 The effect is more pronounced than that due only to the influence of recoil implantation.

\section{HIGH-DOSE IMPLANTATION INTO COMPOUND TARGETS}

Another approach to achieving high concentration of implanted species is to start with a multi-elemental target and then implant one of the elements. This approach is not new and has been used in the case of carbon implantation of $\mathrm{NbC}$ to change the superconductivity transition temperature ${ }^{29}$ For the case of implantation of atomic species $A$ into the mixture $A B$, the situation is nearly the same as for $A$ into $B$ except there is a different boundary condition at steady state. ${ }^{16}$ Instead of $J_{A}=J_{i}$ [Eq. (2)] we now have $J_{A}-J_{B}=J_{i}$. From this relation we can then determine the steady-state surface composition to be

$$
N_{A} / N_{B}=r(S+1) /(S-1)
$$

As an example, we treat the implantation of Si into PtSi. One might anticipate that this approach could result in a very Si-rich Pt-silicide mixture. However, instead of increasing the $\mathrm{Si}$ concentration, the implantation of $\mathrm{Si}$ into the compound may even result in a decrease of the Si concentration because of the preferential sputtering of Si. The composition in the implanted layer will be determined by a competition between implantation and preferential sputtering. The steady-state surface concentration is given by Eq. (8) with $r=1 / 2$ in this case, because sputtering tends to deplete the surface of $\mathrm{Si}$. We plot the ratio of $N_{\mathrm{Si}}$ to $N_{\mathrm{Pt}}$ vs $S$ in Fig. 8. For $S$ greater than 3 , the implanted $\mathrm{Pt}$-silicide sample becomes depleted of $\mathrm{Si}$, because $S$ is sufficiently large so that not enough of the implanted $\mathrm{Si}$ atoms can stay in the sample to overcome the preferential sputtering of $\mathrm{Si}$. For $S=3$, for every incident $\mathrm{Si}$ 


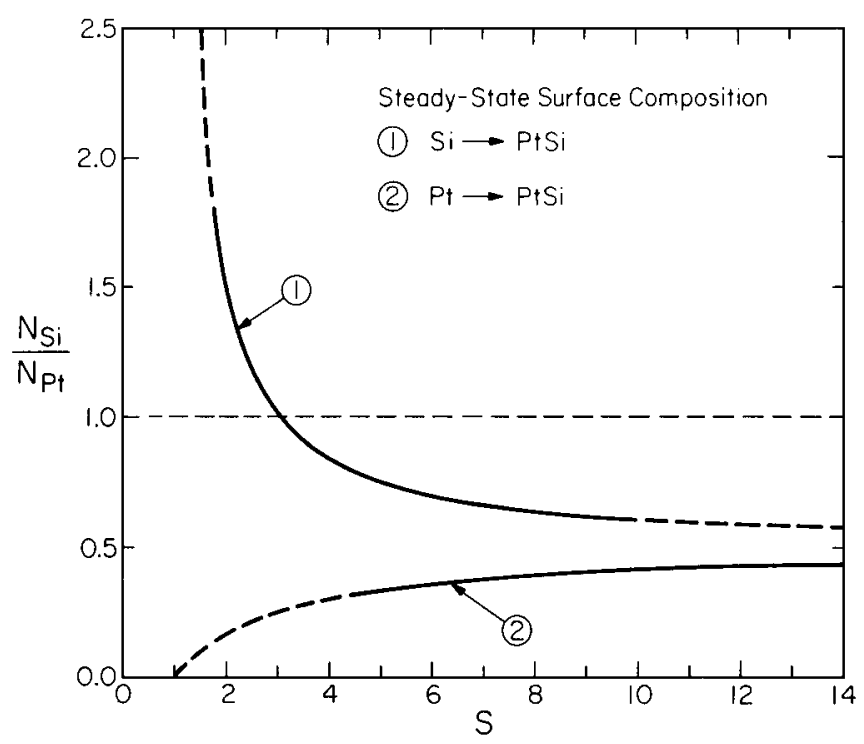

FIG. 8. The predicted steady-state surface composition as a function of total sputtering yield for the implantations of (1) Si and (2) Pt into PtSi. The prediction is based on the preferential sputtering of $\mathrm{Si}$, (i.e. $S_{\mathrm{Si}} / S_{\mathbf{P t}}=2 \cdot N_{\mathrm{Si}} / N_{\mathrm{Pt}}$ ) observed in the inert gas ion sputtering of $\mathrm{PtSi}$.

ion, one $\mathrm{Pt}$ and two Si atoms will be sputtered from the surface layer; the composition of the implanted $\mathrm{Pt}$-silicide sample remains unchanged. For $S<3$, the sample becomes $\mathrm{Si}$ rich. Since $S \simeq 5$ for Ar sputtering of Pt-silicide at $20 \mathrm{keV}$, it seems doubtful that $S<3$ could be achieved in the sputtering of $\mathrm{Pt}$-silicide with Si, except at very high or very low ion energies.

For the same Pt-silicide sample we plot the concentration ratio of $N_{\mathrm{Si}}$ to $N_{\mathrm{Pt}}$ for the case of Pt implantation. The curves in Fig. 8 indicate that in cases where preferential sputtering is dominant, such as mixtures with one light and heavy element, it will be relatively difficult to achieve high concentration of the light (more easily sputtered) species. On the other hand when preferential sputtering does not play a large role, the maximum attainable composition would be determined by the sputtering yield.

\section{CONCLUSIONS}

The maximum achievable concentration of an implanted species is determined in the limit by sputtering or the erosion of the implanted surface. As a rule of thumb one can estimate that the maximum concentration will be proportional to $r / S$ where $S$ is the total sputtering yield and $r$ is the preferential sputtering factor. Since lower mass elements tend to be preferentially sputtered, one can achieve a higher concentration of heavy elements than of lighter elements in the substrate.

As another approach we considered the change in the composition of a compound target implanted with one of the elements in the target. Again preferential sputtering is a major factor and it appears very difficult to achieve high concentrations of preferentially sputtered elements for cases where sputtering yield is above values of 2 or 3 .

Sputtering is a surface phenomena with the sputtered atoms being ejected in the outer most atomic layers of the sample. We have found that atomic mixing can translate changes in the surface composition into depths in the sample comparable to the range of the implanted species. One must consider the width $W$ of the implanted layer as another parameter to be considered when evaluating the influence of high-dose implantation in materials.

The present discussion did not consider any of the practical aspects that often dominate high-dose implantation such as precipitation and cone formation. These factors can lead to changes in the limits presented here. For example it is possible to form oxide layers by implantation of oxygen ions. ${ }^{30}$

One of the major advantages of ion implantation is external control of the number of implanted ions. In high-dose implantation this advantage is lost to good measure due to the dominance of sputtering. However, there are three approaches to achieve unique metallurgical structures by ion bombardment that skirts the limits imposed by sputtering:

(1) use of light ions such as oxygen or carbon for which the sputtering yield is less than unity,

(2) prepare targets close to the desired composition and implant up to 10 at. \%,

(3) deposit a layer of material $A$ on substrate $B$ and rely on atomic mixing during bombardment to form mixtures of $A$ and $B$.

\section{ACKNOWLEDGMENTS}

We acknowledge the help and insight provided in discussions with colleagues at Caltech (B. Y. Tsaur, S. Matteson, and G. Chapman) and at Bell Labs (J. Poate and W. Brown). Financial support was provided in part by the Army Research Office.

${ }^{1}$ A. W. Czanderna, editor, Methods of Surface Analysis (Elsevier, New York, 1975).

${ }^{2} \mathrm{~J}$. M. Poate, K. N. Tu, and J. W. Mayer, editors, Thin Films-Interdiffusion and Reactions (Wiley, New York, 1978).

${ }^{3} \mathrm{~J}$. P. Thomas and A. Cachard, editors, Material Characterization Using Ion Beams, (Plenum, New York, 1978).

${ }^{4} \mathrm{~J}$. W. Mayer and E. Rimini, editors, Ion Beam Handbook for Material Analysis (Academic, New York, 1978).

${ }^{5} \mathrm{G}$. Dearnaley in New Uses of Ion Accelerators, J. F. Ziegler, editor (Plenum, New York, 1975), Chap. 5.

${ }^{6} \mathrm{~S}$. M. Myers in Thin Films-Interdiffusion and Reactions, J. M. Poate, K. N. Tu, and J. W. Mayer, editors (Wiley, New York, 1978), Chap. 14.

${ }^{7}$ G. Carter and J. S. Colligon, Ion Bombardment of Solids, (Elsevier, New York, 1968).

${ }^{8}$ P. D. Towsend, J. C. Kelly, and N. E. W. Hartley, Ion Implantation, Sputtering and Their Applications, (Academic, London, 1976).

${ }^{9} \mathrm{G}$. K. Wehner in Methods of Surface Analysis, A. W. Czanderna, editor (Elsevier, New York, 1975), Chap. 1.

${ }^{10}$ G. Carter, J. S. Colligon, and J. H. Lesk, Proc. Phys. Soc. 79, 299 (1962).

${ }^{11}$ W. K. Chu, J. K. Howard and R. F. Lever, J. Appl. Phys. 47, 4500 (1976).

${ }^{12}$ J. M. Poate, W. L. Brown, R. Homer, W. M. Augustyniak, J. W. Mayer, K. N. Tu, and W. F. van der Weg, Nucl. Instrum. Methods 132, 345 (1976).

${ }^{13}$ Z. L. Liau, W. L. Brown, R. Homer, and J. M. Poate, Appl. Phys. Lett. 30, $626(1977)$

${ }^{14}$ Z. L. Liau, J. W. Mayer, W. L. Brown, and J. M. Poate, J. Appl. Phys. (in press).

${ }^{15}$ R. Kelly, Nucl. Instrum. Methods 149, 553 (1978).

${ }^{16} \mathrm{Z}$. L. Liau and J. W. Mayer, Ion Implantation in Material Science and Technology, J. K. Hirvonen, editor (Academic, New York, 1979), Ch. 2. 
${ }^{17}$ P. Sigmund, Phys. Rev. 184, 383 (1969).

${ }^{18}$ H. H. Andersen and H. L. Bay, Radiat. Eff. 19, 139 (1973).

${ }^{19}$ H. H. Andersen and H. L. Bay, J. Appl. Phys. 46, 2416 (1975).

${ }^{20}$ Z. L. Liau, B. Y. Tsaur, J. W. Mayer, W. L. Brown, and J. M. Poate (unpublished).

${ }^{21}$ W. F. van der Weg, D. Sigurd, and J. W. Mayer, Applications of Ion Beams to Metals, S. T. Picraux, E. P. EerNisse, and F. L. Vook, editors (Plenum, New York, 1974), p. 209.

${ }^{22}$ J. M. Poate and T. C. Tisone, Appl. Phys. Lett. 24, 391 (1974).

${ }^{23}$ R. A. Kant, S. M. Myers, and S. T. Picraux, Ion Implantation in Semiconductors, 1976, F. Chernow, J. A. Borders, and D. K. Brice, editors (Plenum, New York, 1977), p. 191.
${ }^{24}$ P. Revesz, M. Wittmer, J. Roth, and J. W. Mayer, J. Appl. Phys. (in press).

${ }^{25}$ Z. L. Liau and T. T. Sheng, Appl. Phys. Lett. 32, 716 (1978).

${ }^{26}$ J. K. Hirvonen, J. M. Poate, Z. L. Liau, and J. W. Mayer, (unpublished).

${ }^{27}$ E. Arminen, A. Fontell, and V. K. Lindross, Phys. Status Solidi (a) 4, 663 (1971).

${ }^{28}$ W. Wach and K. Wittmaack, Nucl. Instrum. Methods 149, 259 (1978).

${ }^{29} \mathrm{O}$. Meyer in New Uses of Ion Accelerators, J. F. Ziegler, editor (Plenum, New York, 1975), Ch. 6.

${ }^{30} \mathrm{E}$. Giani, D. K. Murti, and R. Kelly in Thin Film Phenomena-Interfaces and Interaction, J. E. E. Baglin and J. M. Poate, editors (Electrochem. Soc., Princeton, 1978), p. 443 